\title{
Assessment of Torque Components in Brushless Permanent-Magnet Machines Through Numerical Analysis of the Electromagnetic Field
}

\author{
Dan M. Ionel, Senior Member, IEEE, Mircea Popescu, Senior Member, IEEE, Malcolm I. McGilp, \\ T. J. E. Miller, Fellow, IEEE, and Stephen J. Dellinger
}

\begin{abstract}
For the calculation of torque in brushless (BL) alternating current motors a local method is proposed, based on the Maxwell stress theory and the filtered contributions due to the harmonics of the magnetic vector potential in the motor air gap. By considering the space fundamental field only, the method can efficiently estimate the average synchronous torque for a variety or motor topologies, including concentrated winding designs. For BL direct current motor analysis a global method is introduced, based on the virtual work principle expressed in terms of energy components in various motor regions. The method leads to simplifications in the average torque calculation and enables the direct identification of the cogging and ripple components. The mathematical procedures have been validated against experiments and other numerical techniques.
\end{abstract}

Index Terms-Brushless (BL) permanent-magnet (PM) motor, cogging torque, electric machine simulation, finite-element analysis (FEA), ripple torque, synchronous machine.

\section{INTRODUCTION}

$\mathbf{T}$ HE problem of accurate and efficient computation of the torque, based on the results of electromagnetic field finiteelement analysis (FEA), was widely studied and a large number of numerical procedures have been proposed by other authors, e.g., [1]-[6]. Most of the techniques are generally applicable to electromagnetic devices and neither exploit the particularities of rotating electrical machines nor provide the kind of insight into the motor parameters and performance, which would directly help with the selections involved in a practical design process.

Other FE methods were especially developed for electrical machines, e.g., [7]-[9], and, on this subject, the present paper brings in further original contributions by proposing two new formulations, one local, based on a version of the Maxwell stress applied to the magnetic field in the motor air gap and, another one global, derived from the variation of the magnetic energy

Paper IPCSD-05-037, presented at the 2004 Industry Applications Society Annual Meeting, Seattle, WA, October 3-7, and approved for publication in the IEEE TRANSACTIONS ON INDUSTRY APPLICATIONS by the Electric Machines Committee of the IEEE Industry Applications Society. Manuscript submitted for review October 22, 2004 and released for publication May 9, 2005.

D. M. Ionel is with the Corporate Technology Center, A. O. Smith Corporation, Milwaukee, WI 53224-9512 USA (e-mail: dionel@ aosmith.com).

M. Popescu, M. I. McGilp, and T. J. E. Miller are with the SPEED Laboratory, Department of Electrical Engineering, University of Glasgow, Glasgow, G12 8LT, U.K. (e-mail: mircea@elec.gla.ac.uk; mal@elec.gla.ac.uk; t.miller@elec.gla.ac.uk).

S. J. Dellinger is with the Electrical Products Company, A. O. Smith Corporation, Tipp City, OH 45371-1899 USA (e-mail: sdellinger@ aosepc.com).

Digital Object Identifier 10.1109/TIA.2005.853377 stored in different motor regions. The two methods have been developed in conjunction with and as an aid to the design and optimization of some innovative motor constructions, but the procedures are generally applicable to brushless (BL) permanent-magnet (PM) motors and, for best demonstration, typical examples of conventional machines are employed throughout the paper. The application to the study of $\mathrm{BL}$ alternating current (BLAC) and BL direct current (BLDC) motors, respectively, is discussed both from a computational efficiency point of view, a minimum number of FE solutions being sought, and from a motor engineering point of view, the assessment of various torque components being accomplished.

\section{BLAC MOTORS}

\section{A. Maxwell Stress Harmonic Filter (HFT) Method}

The electromagnetic torque $T_{e}$ in the motor air gap, on a closed surface of radius $r$, can be calculated by integrating the Maxwell stress tensor. For two-dimensional electromagnetic field models

$$
T_{e}=\frac{\ell_{F e}}{\mu_{o}} \int_{0}^{2 \pi} r^{2} B_{r} B_{\theta} d \theta
$$

where $B_{r}$ and $B_{\theta}$ are the radial and tangential components of the flux density and $\ell_{F e}$ is the active axial length of the electrical machine.

First-order triangular elements are used in the common practice of electromagnetic FEA, in order to ensure the highest computational speed. Satisfactory accuracy is achieved in this case for the magnetic vector potential $A$, while the flux density solution $B$ is an order less accurate, since it is obtained by differentiating the trial functions of $A$

$$
B_{r}=\frac{\partial A}{r \partial \theta} \quad B_{\theta}=-\frac{\partial A}{\partial r} .
$$

Because $A$ is described by a linear function over each finite element, the components of $B$ are piecewise constant within each element and significant errors can arise in the calculation of $B_{\theta}$, especially in the elements adjacent to an interface between different materials [2].

To improve the accuracy of the Maxwell stress calculation, an equivalent formulation of (1), as an explicit function of $A$, will be derived. Within the motor air gap, we define two concentric 


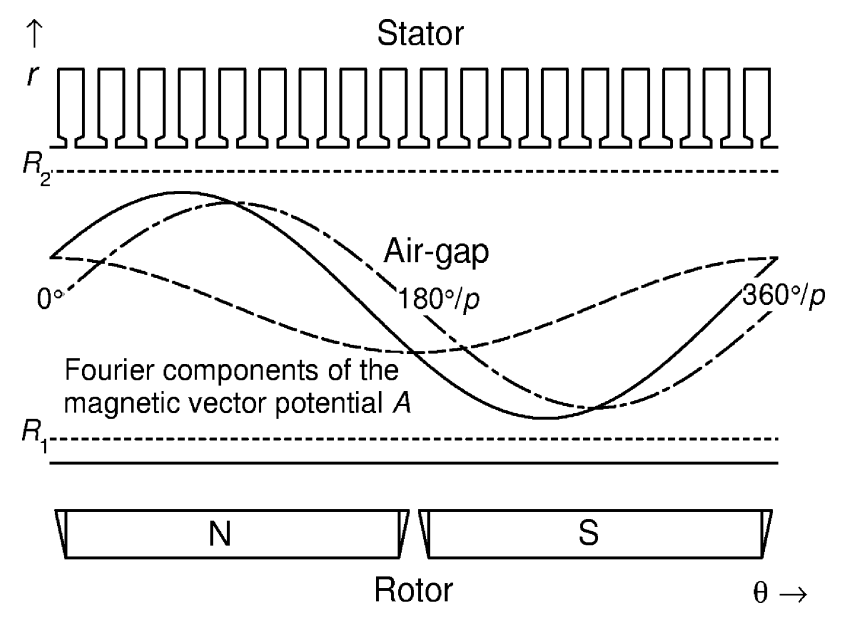

Fig. 1. Explicative for the Maxwell HFT method for computing torque based on the harmonics of the magnetic vector potential in the motor air gap.

circles of radius $R_{1}$ and $R_{2}$, respectively, and force on them nonhomogenous Dirichlet boundary conditions, with the values of $A$ determined by the FEA solution. The main elements, involved in the computations performed on a pair of poles, are schematically represented in Fig. 1, with the fundamental magnetic potential waveform exemplified and plotted with continuous line and its sine and cosine components plotted with dotted lines.

The magnetic vector potential within the cylindrical shell delimited by $R_{1}$ and $R_{2}$ (with $R_{1}<R_{2}$ ) can be analytically described in cylindrical coordinates as [1]

$$
A(r, \theta)=\sum_{n=1}^{\infty}\left(c_{n} r^{n}+d_{n} r^{-n}\right) \cdot\left[g_{n} \cos (n \theta)+h_{n} \sin (n \theta)\right]
$$

The magnetic vector potential on the two circular boundaries can be expressed in Fourier series

$$
\begin{aligned}
& A\left(R_{1}, \theta\right)=a_{01}+\sum_{n=1}^{\infty}\left[a_{n 1} \cos (n \theta)+b_{n 1} \sin (n \theta)\right] \\
& A\left(R_{2}, \theta\right)=a_{02}+\sum_{n=1}^{\infty}\left[a_{n 2} \cos (n \theta)+b_{n 2} \sin (n \theta)\right]
\end{aligned}
$$

and, by writing (3) for $R_{1}$ and $R_{2}$, the cross products of the coefficients are identified as

$$
\begin{aligned}
& c_{n} g_{n}=\frac{1}{\delta_{n}} \cdot\left[a_{n 1}\left(\frac{R_{1}}{R_{2}}\right)^{n}-a_{n 2}\right] \\
& c_{n} h_{n}=\frac{1}{\delta_{n}} \cdot\left[b_{n 1}\left(\frac{R_{1}}{R_{2}}\right)^{n}-b_{n 2}\right] \\
& d_{n} g_{n}=\frac{1}{\delta_{n}} \cdot\left[a_{n 2}-a_{n 1}\left(\frac{R_{2}}{R_{1}}\right)^{n}\right] \\
& d_{n} h_{n}=\frac{1}{\delta_{n}} \cdot\left[b_{n 2}-b_{n 1}\left(\frac{R_{2}}{R_{1}}\right)^{n}\right]
\end{aligned}
$$

where

$$
\delta_{n}=\left(\frac{R_{1}}{R_{2}}\right)^{n}-\left(\frac{R_{2}}{R_{1}}\right)^{n}
$$

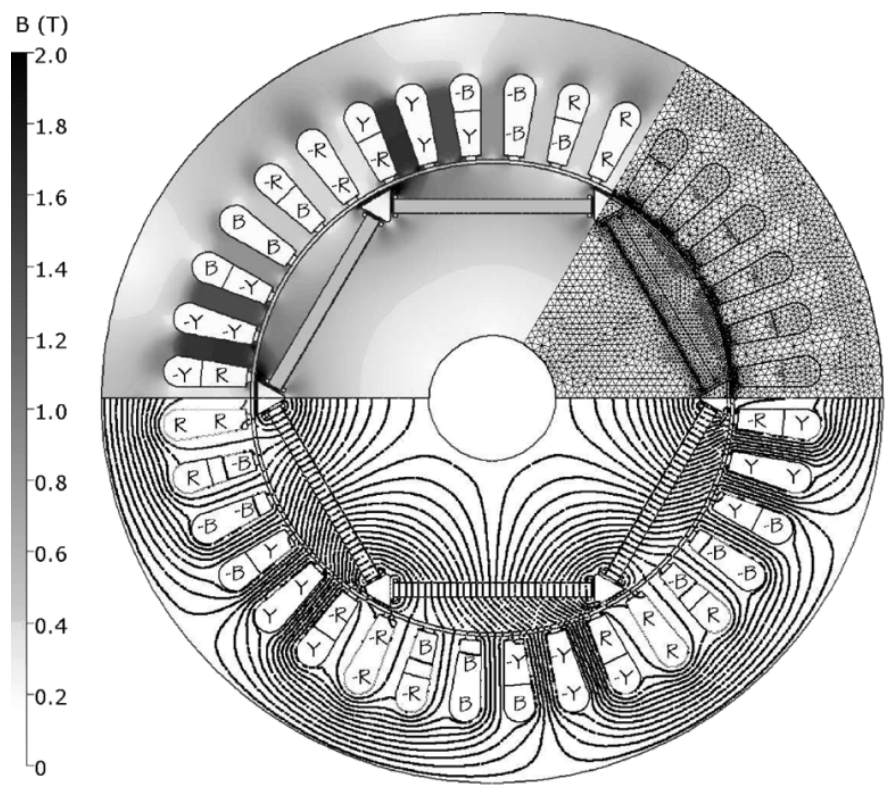

Fig. 2. FE model of a six-pole IPM motor prototype with three-phase short-pitched distributed windings.

and it has been taken into account the fact that in balanced BLAC machines without homopolar flux, the DC components $a_{01,2}$ are equal to zero.

Substituting (6)-(10) in (3), employing discrete formulations for (1), (2), and considering the symmetries present in a motor with $p$ pole pairs, a new computational formula is derived for the electromagnetic torque

$$
T_{e}=p \frac{2 \pi \ell_{F e}}{\mu_{o}} \sum_{n=1}^{\infty} \frac{n^{2}\left(a_{n 2} b_{n 1}-a_{n 1} b_{n 2}\right)}{\left(\frac{R_{1}}{R_{2}}\right)^{n}-\left(\frac{R_{2}}{R_{1}}\right)^{n}}
$$

Most notably, this equation illustrates the mechanism of torque production by the interaction of same-order air-gap field harmonics. A certain harmonic will provide a nonzero torque contribution if the sine and/or cosine coefficients of the magnetic vector potential waves on the two boundaries are different, respectively. Physically, this would be caused by a change in the field direction, i.e., a variation of the radial and tangential flux density components, under the provisions of the Gauss law of magnetic flux conservation.

Other authors [3]-[5], using different approaches based on the coefficients $c_{n}, d_{n}, g_{n}$, and $h_{n}$ of (3), have developed formulas equivalent, in principle, with (11). Our approach, beyond its advantage of a simpler and easier to implement formulation, which can be used in conjunction with any general-purpose electromagnetic FEA software package, also enables insights into the torque components of a BLAC motor.

The method based on (11), which resembles a harmonic filter and is, therefore, referred to as Maxwell HFT, has been implemented in the scripting language of an FEA software package [10] and was validated on a number of example prototype BL PM motors manufactured especially for this purpose. A BLAC motor of the interior PM (IPM) type (Fig. 2) was built with the stator lamination of a 184-frame three-phase induction motor. The 36-slot core was wound with a 5/6 short-pitched double- 


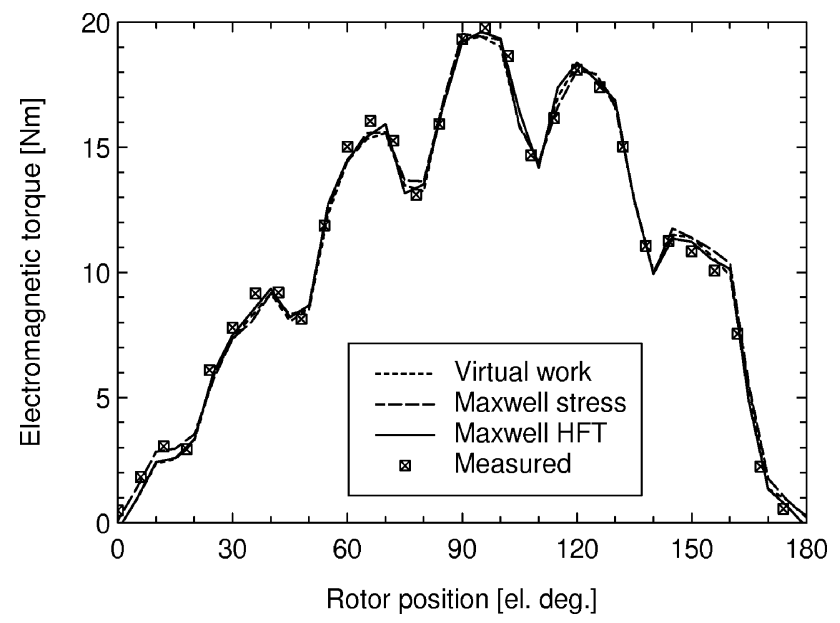

Fig. 3. Experimental and computed standstill (static) torque for the BLAC IPM prototype motor of Fig. 2. (The prototype has no rotor-stator axial skew.)

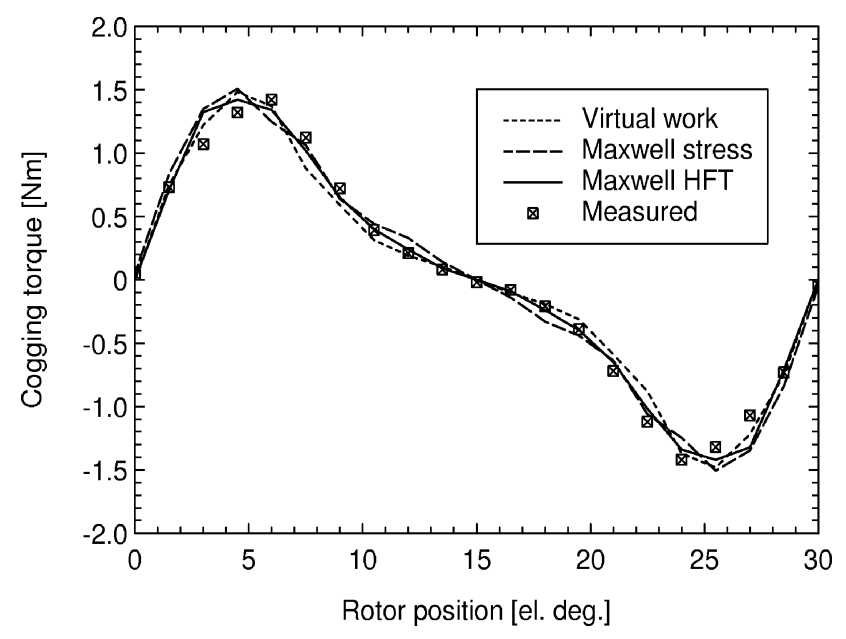

Fig. 4. Experimental and computed cogging torque of the BLAC IPM prototype motor of Fig. 2.

layer distributed winding. The rotor was made using rectangular PM blocks of $\mathrm{NdFeB}$. In order to enable a fair comparison of measured and FEA results, the prototype has no rotor-stator axial skew and was tested at standstill (statically) with DC current excitation, equivalent to an instantaneous AC current distribution having the current in two phases equal to half the value, and the opposite direction of the current in the third phase. The results of this test at 15-A DC source current (Fig. 3), as well as those of the cogging measurements (Fig. 4) indicate satisfactory agreement of the Maxwell HFT results with the experimental data, as well as with two other conventional and well established numerical methods, namely, the Maxwell stress [2] and the virtual work as per Coulomb-Meunier special FEA formulation [6].

From a computational point of view, the Maxwell HFT method is advantageous due to its reduced sensitivity to finite element meshing, especially in the air gap. On the other hand, the Maxwell HFT method is sensitive to parameters such as the maximum order of the harmonics considered for summation. For example, only 71 harmonics were required for the convergence of the electromagnetic torque calculation of Fig. 3 and

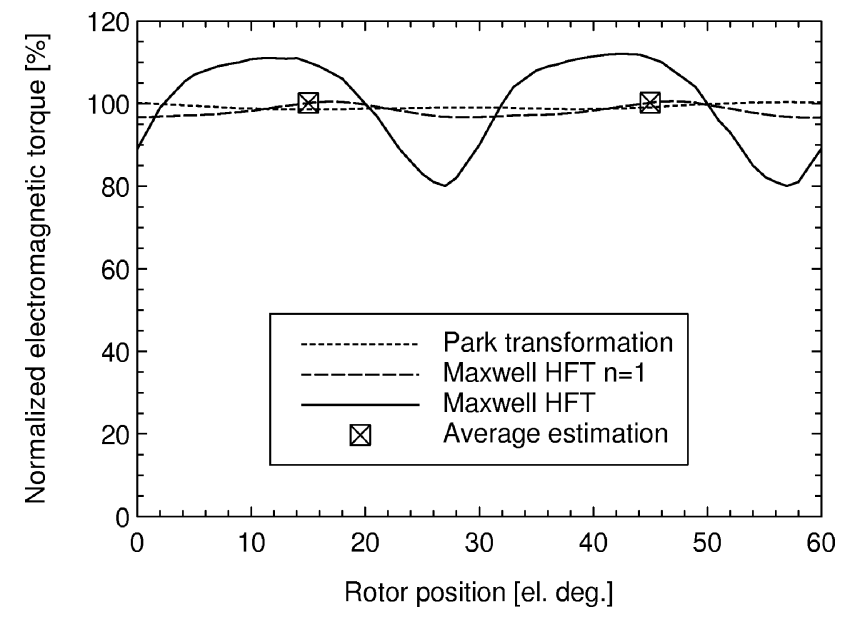

Fig. 5. Computed electromagnetic torque versus rotor position for the prototype IPM motor of Fig. 2 operating at rated load.

a larger number of 151 harmonics was needed for the cogging torque calculations of Fig. 4. Such numerical aspects have been discussed for example in [4] and [5]. The following sections are concentrated on aspects related to the torque components in a BLAC motor, which have not been previously studied in conjunction with the Maxwell HFT numerical method.

\section{B. Average Synchronous Torque Estimation}

The resultant rotating magnetic field under load operation is determined by both the rotor permanent magnetization and the stator armature currents and has a space fundamental component, which travels at synchronous speed in the motor air gap and is responsible for the production of the average torque. The open-circuit flux density high-order space harmonics produced by the rotor magnetization also travel at synchronous speed, but their contribution to average torque is zero because their polarity is different to that of the fundamental space harmonic of the armature magnetomotive force (MMF), which is the only one of the stator harmonics that rotates at synchronous speed.

The combination of this rationale with the Maxwell HFT formulation indicates that the average torque component can be estimated by limiting the summation in (11) to the first electrical harmonic only. Fig. 5 shows the results of such a numerical exercise for the FEA model of the IPM motor of Fig. 2 running synchronously at rated current and a torque angle of $110^{\circ}$. The stator currents have been considered to be purely sinusoidal (free of switching ripple) and the motor operation was simulated through a succession of magnetostatic "snap-shots." The total electromagnetic torque, inclusive of harmonic ripple components, has been calculated with (11) and $n=71$. The torque has also been evaluated with the $d q$ formulation

$$
T_{e}=\frac{3}{2} p\left(\Psi_{d} i_{q}-\Psi_{q} i_{d}\right)
$$

where the $d q$ flux linkages $\Psi_{d, q}$ and currents $i_{d, q}$ have been determined at each rotor position by applying the Park transformation to the phase quantities.

The torque curve calculated by the Maxwell HFT with $n=1$ contains a relatively small peak to peak ripple of $3.4 \%$, which is comparable with the ripple of the torque computed with the $d q$ 
equation (11). Several factors should be considered in the explanation of this torque ripple. As the rotor moves, the air-gap permeance varies and the PM operating point oscillates around an average value, a phenomenon that has been studied for example in [8] in relation with the cogging torque production. As a consequence, the equivalent MMF of the PM and the fundamental air-gap field vary and the rotor positions of zero cogging are more likely to provide the basis for average torque estimation. The motor magnetic circuit is shared by the stator armature field and local saturation can also play a role in the oscillations of the average torque calculations. Of the two types of zero-cogging position, the one corresponding to the alignment of the rotor inter-pole axis with the center of a slot opening (15 electrical degrees for this example motor) not only enjoys a much smaller derivative of the cogging torque but also exhibits a favorable alignment of the stator MMF.

For this example motor, the torque calculated with Maxwell HFT and $n=1$ at 15 and 45 electrical degrees, has been found to be less than $1 \%$ different of the average value of the total electromagnetic torque. The effect of saturation on the estimation has been examined by simulating motor operation between $25 \%-200 \%$ of rated load and agreement within $1 \%$ has been observed.

The comparison of the Maxwell HFT equation (11), written for the contribution of the fundamental wave only, and of (12), which is based on the $d q$ theory, points out a similarity in that waveforms placed in quadrature are responsible for torque production. Unlike the $d q$ theory, which identifies the sources of torque as flux linkages and currents, the Maxwell HFT method indicates as the torque source, the spatial shift of the total magnetic field along the air-gap radius.

From a theoretical point of view, the $d q$ theory is confined to perfectly sinusoidally distributed windings and excitation. While for the IPM motor of Fig. 2 this could be an acceptable engineering assumption, it is certainly not valid for BLAC motors with concentrated windings - a design that is gaining large popularity in industry. Such a three-phase six-pole example with nine slots and with coils wound around each tooth is shown in Fig. 6. This motor, although it exhibits a rather large harmonic content, still has a rotating magnetic field in the air gap and the considerations regarding the calculation of average torque with the Maxwell HFT method, which were stated in the analysis of the previous example, are valid. The FEA results from Fig. 7 show that in this case the Maxwell HFT method with $n=1$ has a smaller ripple than the $d q$ approximation and also provides a good estimation, within $1 \%$, of the average torque in the most favorable positions of 0 and 60 electrical degrees. Again, the estimation of the average torque maintains a satisfactory precision, within $2 \%$ for loads between $25 \%-200 \%$ of rated value. Other motor designs, with concentrated windings, three slots per each pole pair, different polarity and electromagnetic loading, have been simulated with satisfactory results.

The method of average torque estimation, with the Maxwell HFT equation applied only for the fundamental magnetic vector potential waveform, is remarkable, as it requires only one FE solution at a suitably selected rotor position and current distribution. This can be extremely useful, especially in the early stages of designing a new motor, when computational speed is of the essence.

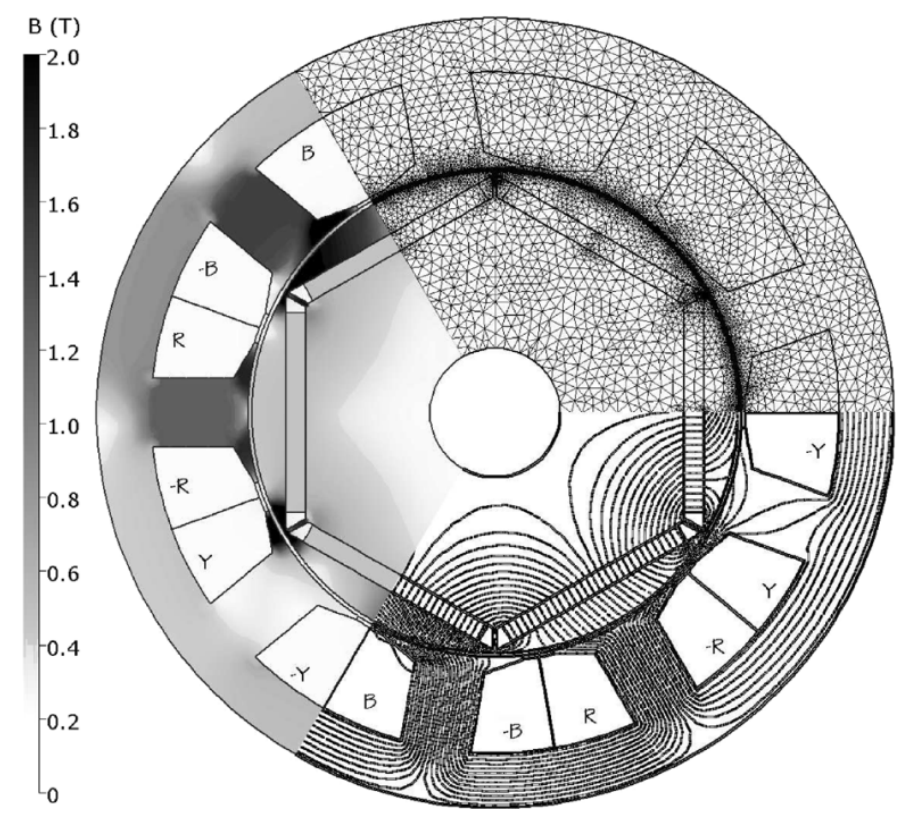

Fig. 6. FE model of a six-pole IPM motor design with three-phase concentrated windings. One pole pair is used in the simulations.

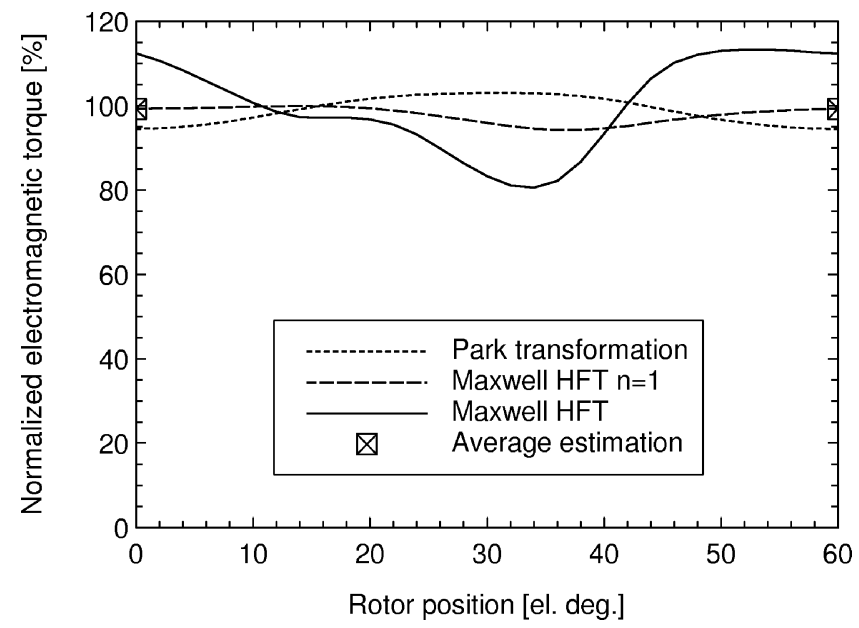

Fig. 7. Computed electromagnetic torque versus rotor position for the IPM motor design of Fig. 6 operating at rated load.

\section{Pulsating and Average Torque Components}

The Maxwell HFT method can also be used in conjunction with the "frozen" permeability technique [11], which consists of nonlinearly solving by FEA the electromagnetic field with all sources (PM and currents) present, calculating the value of permeability within each element, and then fixing and using these values for a linear FEA, where only some of the sources are present. Through this approach, the average and ripple torque components [12], [13], associated with various field sources and motor design parameters, can be separated. The results can provide valuable insight into electrical machine optimization, and will be exemplified here using four IPM designs employing the same stator lamination (Fig. 8).

Motors A and D use the same conventional IPM rotor. The rotor of motor B has sinusoidally profiled poles and the rotor of motor $\mathrm{C}$ has slits, which act as $q$-axis flux barriers. The same 

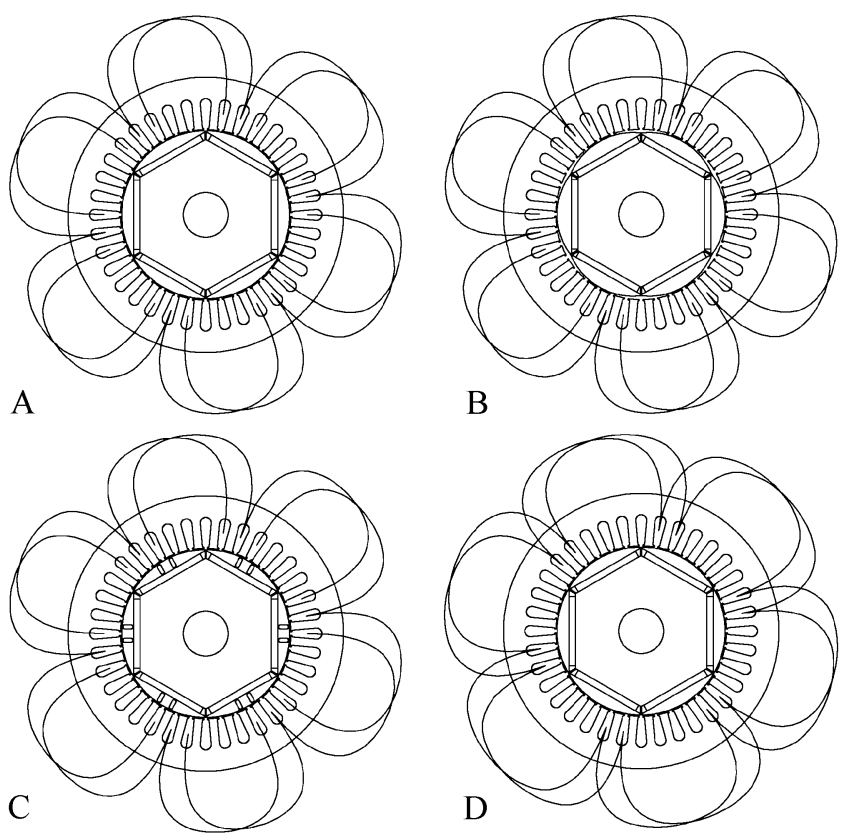

Fig. 8. Four IPM motor designs used for comparison. (a) Conventional rotor and 5/6 short-pitched stator windings. (b) Rotor poles with sinusoidal profile. (c) $q$-axis rotor slits. (d) Conventional rotor and fully pitched stator windings. Only one phase winding is shown.

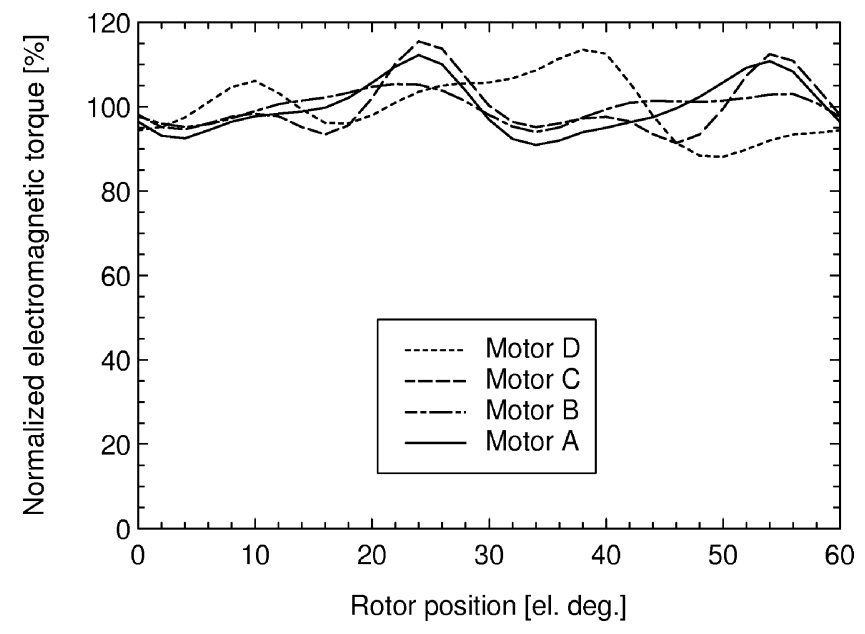

Fig. 9. Computed total electromagnetic torque versus rotor position for the four IPM designs of Fig. 8.

short-pitched winding pattern is employed in all examples, except motor D, which has a fully pitched winding. The magnet length in the direction of magnetization has been maintained and the number of turns has been adjusted so that the motors have approximately the same torque constant. The physical dimensions of the motors and the torque output are comparable with those of the prototype of Figs. 2-4.

The comparative results of the torque components for rated current and a torque angle of $120^{\circ}$ are shown in Figs. 9-11. The largest peak-to-peak ripple of the total electromagnetic torque stands at $28 \%$ and is exhibited by design $\mathrm{D}$, which points to the higher harmonic content of the stator MMF as a major cause. Of the other three designs - in which the fifth and seventh stator MMF harmonics and their multiples are greatly reduced-motor $\mathrm{C}$ has the largest electromagnetic

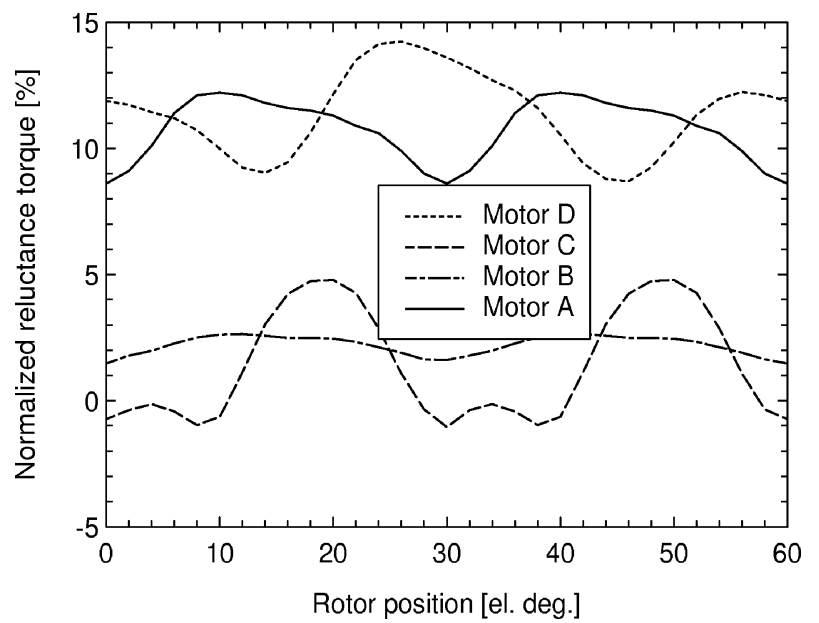

Fig. 10. Computed reluctance torque versus rotor position for the four IPM designs of Fig. 8.

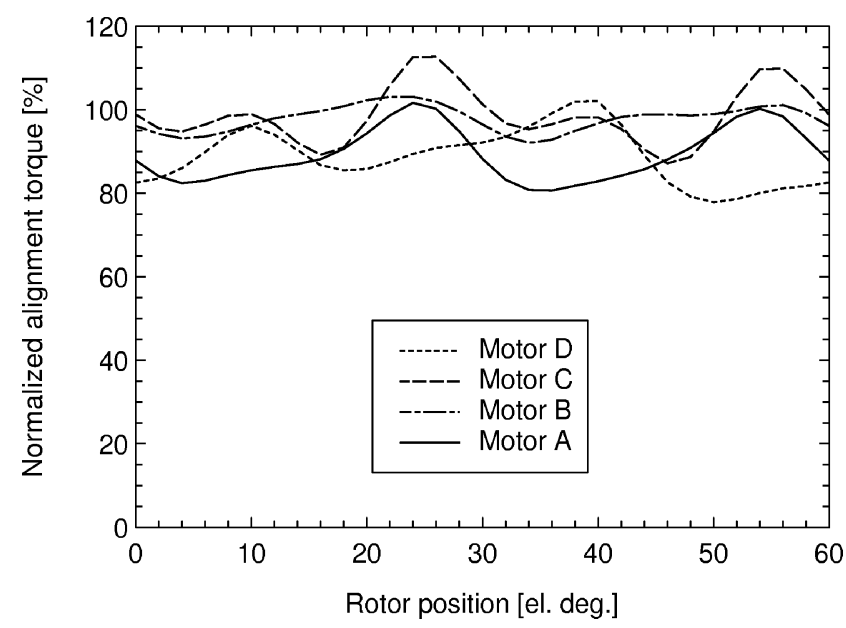

Fig. 11. Computed alignment torque versus rotor position for the four IPM designs of Fig. 8.

ripple caused by the distortion of the field due to the rotor slits, which are placed in the path of the $q$-axis armature flux.

The rotor slits are also responsible for greatly reducing the $d q$ magnetic saliency and therefore the average reluctance torque, as well as for introducing a large ripple into the reluctance torque waveform of Fig. 10. The reluctance torque was computed with the "frozen" permeability technique by setting the PM magnetization to zero. For a real IPM motor, this has resulted in a virtual synchronous reluctance motor, which can be imagined as operating concurrently on the same shaft with another virtual motor, which produces only alignment torque. The alignment torque data of Fig. 11 have been calculated as the difference between the total electromagnetic torque and the reluctance torque.

The modification of the air-gap permeance function, due to rotor pole profiling in design $\mathrm{B}$, has resulted in reduced ripple for all torque components. Also, the equivalent "on-load" cogging torque, which was calculated with the "frozen" permeability technique, by setting the stator currents to zero, has been reduced to approximately half of the value corresponding to the other three motors. However, keeping the global design picture in mind, it should be noted that motor B has its own drawbacks, 
requiring, in order to produce the same output torque, a reduced minimum air gap and/or having increased copper losses.

\section{BLDC MOTORS}

\section{A. Virtual Work Method With Separation of Torque Components ( $\mathrm{SCO}$ )}

According to the virtual work principle, the electromagnetic torque $T_{e}$ equals the derivative of the magnetic co-energy $W_{m}^{\prime}$ with respect to angular position at constant current. Combining this method with the Legendre transformation, which states that in a conservative system the variation of the input energy $W_{\text {in }}$ equals the sum of the variation of magnetic energy $W_{m}$ and magnetic coenergy $W_{m}^{\prime}$, leads to

$$
T_{e}=\left.\frac{\Delta W_{m}^{\prime}}{\Delta \theta}\right|_{i=c t}=\left.\frac{\Delta W_{i n}}{\Delta \theta}\right|_{i=c t}-\left.\frac{\Delta W_{m}}{\Delta \theta}\right|_{i=c t} .
$$

By expressing the input energy into a BLDC motor as a function of the flux linkages $\Psi_{k}$ and currents $i_{k}$ through the coils, and after mathematical manipulations, a formula for the electromagnetic torque developed by an ideally commutated BLDC motor with square-wave currents, which are assumed constant between two commutation switches, is derived as

$$
T_{e}=\left.\sum_{k=1}^{n} \frac{1}{2} \frac{i_{k} \Delta \Psi_{k}}{\Delta \theta}\right|_{i=c t}-\left.\frac{\Delta W_{m l c}}{\Delta \theta}\right|_{i=c t}
$$

where $W_{m l c}$ is the magnetic energy stored in the system less the magnetic energy stored in the coils and includes components from the air gap, magnets, rotor and stator steel and any other motor regions which are not part of the coils. The formulas employed for energy calculations are listed in the Appendix.

The integral over an electrical cycle of the first term from the right-hand side of (14) provides the average torque $T_{\text {avg }}$, as can be demonstrated, for example, with the theory of the flux-MMF diagram [7]. At any given moment in time, this term contains the contributions due to the back-electromotive-force (EMF) harmonics to a ripple torque component, which we define as

$$
T_{r m f}=\left.\sum_{k=1}^{n} \frac{1}{2} \frac{i_{k} \Delta \Psi_{k}}{\Delta \theta}\right|_{i=c t}-T_{\text {avg }} .
$$

At zero current (i.e., open circuit) the second term of (14) is nothing else than the cogging torque, which is due to the variable permeance of the magnetic circuit, and by analogy we define an "on-load" cogging torque

$$
T_{c l d}=-\left.\frac{\Delta W_{m l c}}{\Delta \theta}\right|_{i=c t}
$$

Coulomb proposed a stable method that enables the direct computation of the total electromagnetic torque by a virtual numerical displacement [6], rather than through a derivative as in (15), (16). However, our technique-which enables the separation of the pulsating torques and is therefore referred to as the virtual work SCO method-quantifies the ripple and cogging components and can provide valuable engineering support in a BLDC motor design optimization process.

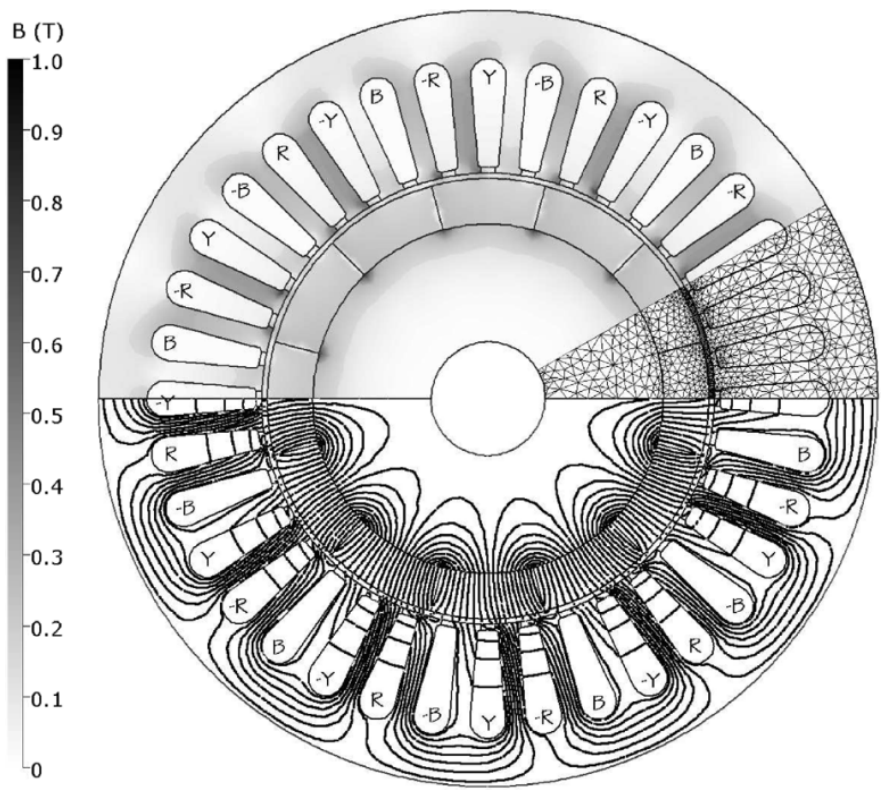

Fig. 12. FE model of a 12-pole BLDC prototype motor with a distributed fully pitched stator winding and a rotor with surface-mounted ferrite arcs.

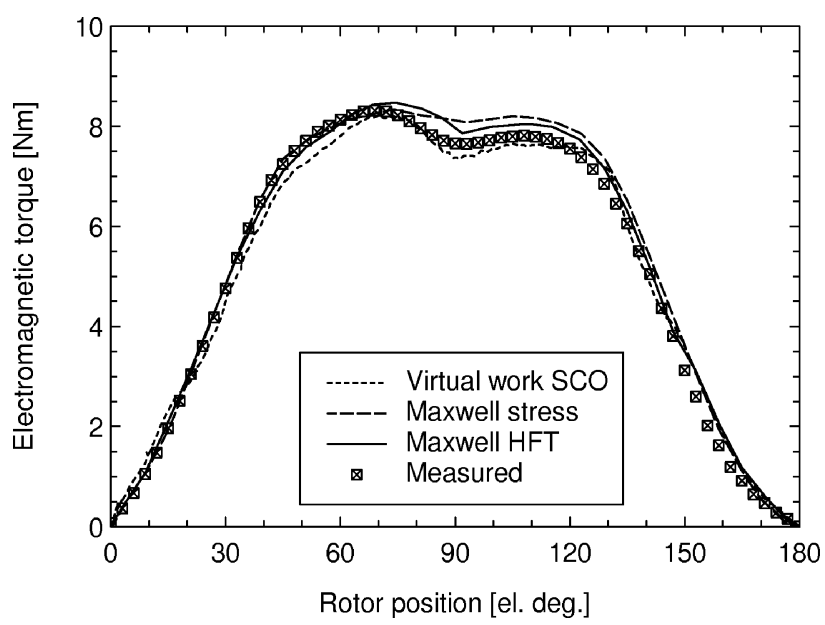

Fig. 13. Experimental and computed standstill (static) torque for the BLDC prototype motor of Fig. 12.

The virtual work SCO method has been implemented in the scripting language of an FEA software [10] and was validated on a number of example prototype brushless PM motors manufactured especially for this purpose. A BLDC motor has been made using the stator core of a 48-frame induction motor, which was wound with a 12-pole fully pitched three-phase winding (Fig. 12). The experimental rotor was built by gluing 12 premagnetized ferrite arcs on a cylindrical core of laminated steel. In order to enable a fair comparison of measured and FEA results, the motor has no stator-rotor axial skew and was tested at standstill with DC current excitation that is representative of BLDC operation, i.e., two phases connected in anti-series and the third left open.

This test and the cogging measurements indicate satisfactory agreement of the results provided by (14), with the experimental data, as well as with the Maxwell stress and the Maxwell HFT 


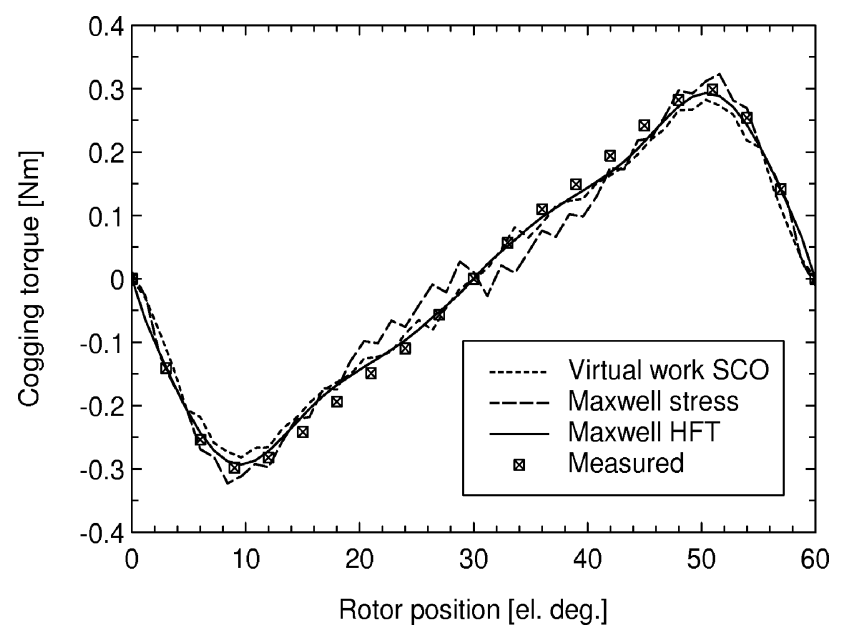

Fig. 14. Experimental and computed cogging torque for the BLDC prototype motor of Fig. 12. (The prototype has no rotor-stator axial skew.)

calculations (Figs. 13 and 14). The results of the cogging torque computation, which is known as a challenging procedure, illustrate the numerical noise-influenced by the combination of mesh discretization and rotation step-which is present in the Maxwell stress output and, to a much lesser extent, in the virtual work SCO results.

The results shown in Figs. 13 and 14 were also compared with those calculated using a method that integrates the Maxwell stress over the entire cross-sectional area of the air gap [14] and which has gained acceptance for practical applications, e.g. [15], [16]. For the particular mesh employed in the numerical example, the differences between the results of this integral method and those of Maxwell HFT calculations were within $2 \%$.

\section{B. Average Torque Calculation With the $i-\Psi V 8$ Diagram}

The current regulated BLDC operation of the IPM motor example of Fig. 2 was simulated and the results have been used to draw the per-phase electrical cycles in the $i-\Psi$ plane of Fig. 15. This example was selected also in order to illustrate the effect of variable reluctance on the operation with square-wave currents. It can be observed that, under the assumptions of ideal BLDC operation (simulated in Fig. 15 with a fixed rotational step of 3 electrical degrees), the diagram can be greatly simplified to a version that requires only eight points and is referred to as $i-\Psi V 8$. The eight points (corners) are clearly marked in Fig. 15 and they correspond to the on/off phase commutation moments (roman numerals: I, II, III, and IV) and to the moments immediately before or after the phase commutation (arabic numerals: 1, 2, 3, and 4). The symmetries for positive and negative current of an $i-\Psi$ phase diagram enable the reduction of information requirements for the simplified version to only four points.

To compute, for example, the half cycle with positive currents, the rotor is positioned where the switching on of the studied phase occurs and an FE mesh is generated. This mesh is solved, considering the PM excitation and the current distribution just before the instant commutation, to calculate the phase

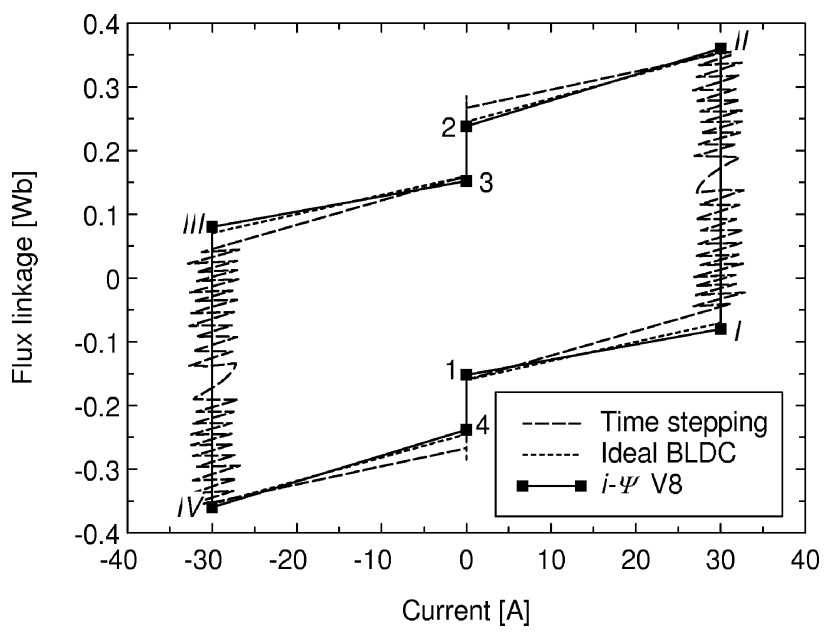

Fig. 15. Example of an $i-\Psi V 8$ diagram for the IPM motor prototype of Fig. 2 operated as a BLDC motor.

flux linkage $\Psi_{1}$, and it is also separately solved, considering the PM excitation and the current distribution just after the instant commutation, to calculate the phase flux linkage $\Psi_{I}$. The rotor is then moved by an angle equal to the phase conduction period, typically 120 eletrical degrees, to the position where the switching off of the studied phase occurs and another FE mesh is generated. This second mesh is solved, considering the PM excitation and the current distribution just before the instant commutation, to calculate the phase flux linkage $\Psi_{I I}$, and it is also separately solved, considering the PM excitation and the current distribution just after the instant commutation, to calculate the phase flux linkage $\Psi_{2}$. This newly described procedure results in remarkable savings of computational effort as only two FE meshes and a total of four FE solutions are required.

It has been previously demonstrated that the area of the flux MMF [7] or $i-\Psi$ diagram [9] is proportional to the average electromagnetic torque. In the case of the simplified $i-\Psi V 8$ diagram, this leads to

$$
T_{\mathrm{avg}}=2 m\left(\frac{\Psi_{I I}+\Psi_{2}}{2}-\frac{\Psi_{I}+\Psi_{1}}{2}\right) I_{S P}
$$

where $m$ is the number of phases and $I_{S P}$ is the set-point phase current.

Further simplification could be possible, in principle, by assuming that the flux linkage difference $\Psi_{I I}-\Psi_{I}$ equals $\Psi_{2}-$ $\Psi_{1}$. In the example calculation of Fig. 15 the electric loading and the magnetic saturation have been selected on purpose to high levels to illustrate that this is not necessarily an acceptable approximation and a per-case judgment should be made. However, (17) is generally valid, under the stated assumption of ideal BLDC square-wave currents, and provides the average electromagnetic torque as function of only the phase flux linkages at the beginning and the end of a conduction period, without any restrictions regarding the shape of the flux linkage or back-EMF waveform. Furthermore, the cycles plotted in Fig. 15 suggest that, due to curve intersection and area compensation, the $i-$ 


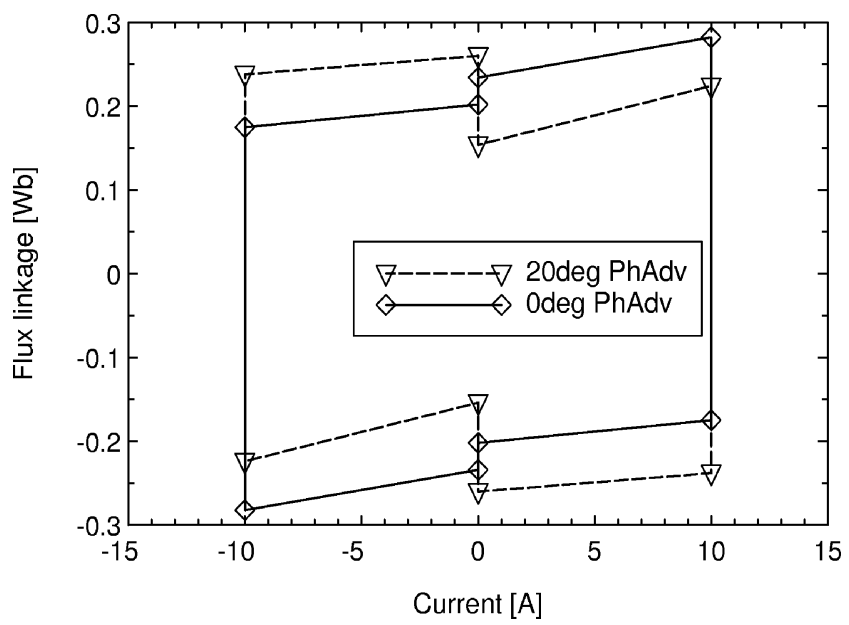

Fig. 16. Effect of phase advance on the $i-\Psi V 8$ diagram for the IPM motor prototype of Fig. 2 operated as a BLDC motor.

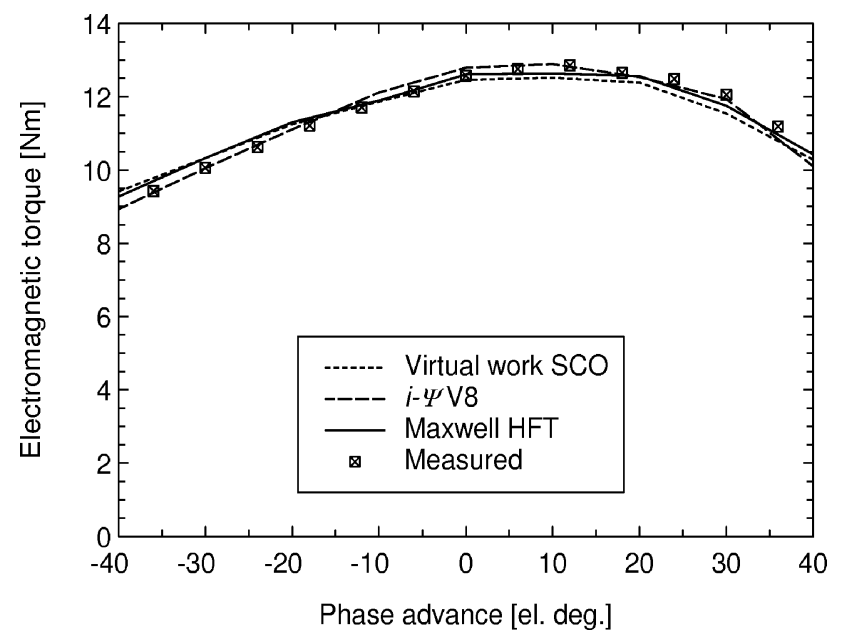

Fig. 17. Experimental and computed standstill (static) average torque versus phase advance for the IPM prototype of Fig. 2 operated as a BLDC motor.

$\Psi V 8$ approximation could compare satisfactorily with the average torque output under realistic current switching motor operation, which is modeled through a significantly more computationally extensive time-stepping procedure.

The graphics of Fig. 16 are selected from a set of computations studying the effect of the phase advance angle on the torque produced by a variable reluctance PM motor, represented by the IPM prototype of Fig. 2, and illustrate the shape and offset changes in the $i-\Psi V 8$ diagram. The average torque calculations have been satisfactorily compared with experimental data, which was collected from an IPM prototype motor with skewed rotor, and with results computed by other methods (Fig. 17).

\section{Direct Calculation of Ripple and Cogging Torque}

Using the average torque calculated with (17), the ripple and the cogging torque components of the pulsating torque, can be directly assessed at any given rotor position with (15) and (14), respectively. The results of such a study for the BLDC prototype of Fig. 12 are displayed in Figs. 18 and 19. The increase in current loading from 6 to 9 A causes a slight reduction of the "on-load" cogging torque, which can be physically explained through a small modification of the equivalent slot opening due

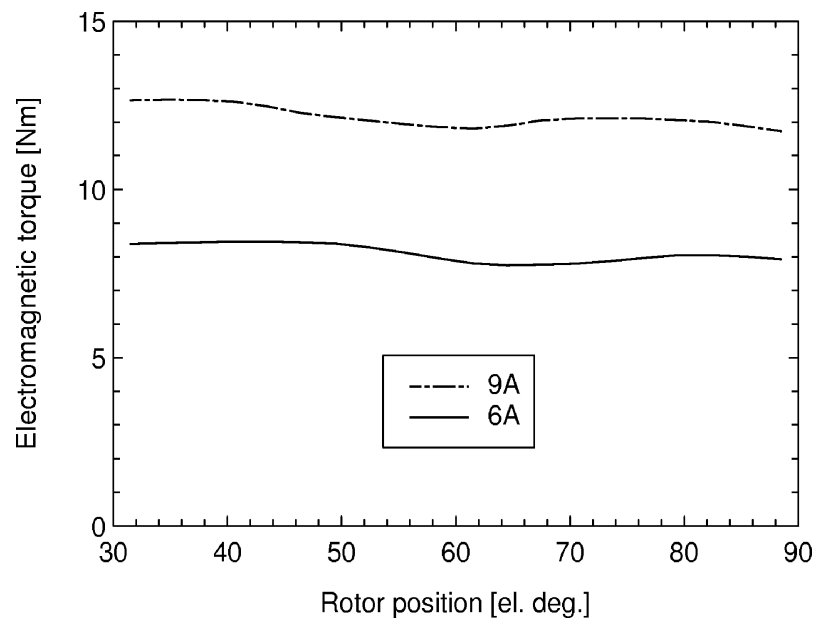

Fig. 18. Total electromagnetic torque computed for the prototype BLDC motor of Fig. 12.

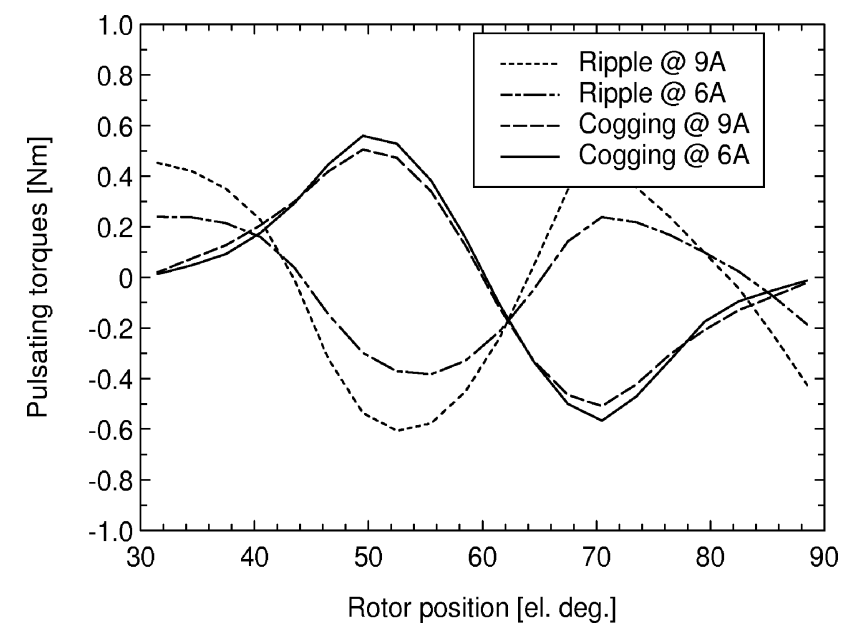

Fig. 19. Cogging and ripple torque components computed for the prototype BLDC motor of Fig. 12.

to tooth tip saturation. On the other hand, at overload, the torque ripple component due to the back-EMF harmonics is increasing in approximately the same ratio as the current loading, an effect related the global saturation of the magnetic circuit. Also interesting to note is the fact that the cogging and the ripple components are close to being in anti-phase, which explains the relatively low peak to peak total ripple observed in the total electromagnetic torque.

Such a nonlinear analysis can be helpful in selecting the most appropriate design features, such as number of slots per pole, winding pattern, "dummy" notches in the stator teeth, rotor magnetization, relative axial skew of rotor to stator, etc, which will mitigate one or both of the pulsating BLDC torque components.

\section{CONCLUSION}

The methods introduced are geared toward the specifics of the electromagnetic field in brushless PM motors. For BLAC motors, the new Maxwell HFT formulation provides better accuracy for a given mesh and, when used in conjunction with the "frozen" permeabilities technique, yields the separation of the 


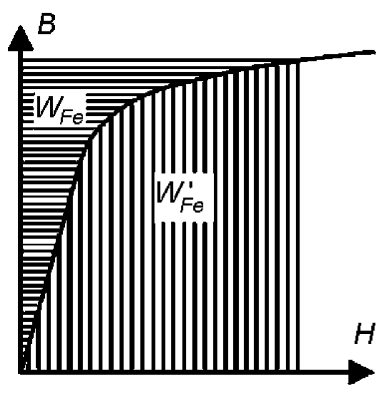

(a)

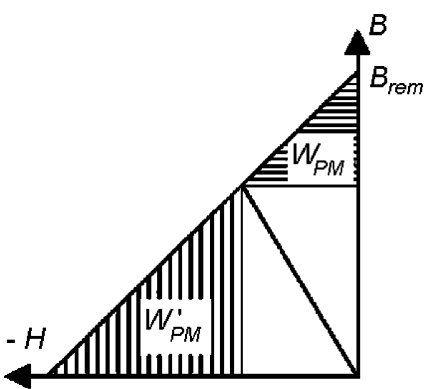

(b)
Fig. 20. Explicative for computing the magnetic energy in (a) soft-magnetic materials (e.g. laminated electric steel) and (b) PMs with a rigid demagnetization characteristic (e.g. rare earth or ferrite).

alignment, reluctance and cogging components out of the total electromagnetic torque. By considering only the contribution of the fundamental wave of the magnetic vector potential in the air gap, and by selecting a rotor position dependent on the motor topology, the average torque can be estimated from a single FE solution, an approach that is beneficial in the early stages of a practical design process when fast computation is of the essence.

For BLDC motors, the new virtual-work SCO formulation ensures the basis of ripple and cogging torque identification and provides engineering support in selecting the design methods to be used for the mitigation of parasitic torque effects. In combination with a simplified version of the $i-\Psi$ diagram, and under the assumptions of ideal BLDC operation, the method can provide an estimation of the average torque with as little as two FE solutions. Due to the global field approach, a coarser mesh can be used for this average torque calculation, resulting in further computational savings and making the method highly suitable for application in an industrial design environment.

\section{APPENDIX}

The volume density of magnetic energy in a domain with a linear $B-H$ characteristic (i.e., constant permeability), such as an air-filled region, is calculated as

$$
w_{a}=\frac{1}{2} B H .
$$

For soft-magnetic materials (e.g., laminated electric steel) the magnetic energy density (Fig. 20) can be estimated by

$$
w_{F e}=\int_{0}^{B} H d B
$$

and in isotropic hard-magnetic materials (i.e., PMs) [17], [18]

$$
w_{\mathrm{PM}}=\frac{1}{2 \mu_{0} \mu_{r p}}\left(B_{p}-B_{r e m}\right)^{2}+\frac{1}{2 \mu_{0} \mu_{r t}} B_{t}^{2}
$$

where $B_{r e m}$ is the remanent flux density, $B_{p}$ and $B_{t}$ are the components of flux density and $\mu_{r p}$ and $\mu_{r t}$ are the relative permeabilities parallel and transversal to the axis of easy magnetization.

\section{REFERENCES}

[1] K. Binns, P. Lawrenson, and C. Trowbridge, The Analytical and Numerical Solution of Electric and Magnetic Fields. Chichester, U.K.: Wiley, 1992.

[2] S. Salon, Finite Element Analysis of Electrical Machines. Boston, MA: Kluwer, 1995.

[3] A. Abdel-Razek, J. Coulomb, M. Feliachi, and J. Sabonnadiere, "The calculation of electromagnetic torque in saturated electric machines within combined numerical and analytical solutions of the field equations," IEEE Trans. Magn., vol. 17, no. 6, pp. 3250-3252, Nov. 1981.

[4] K. Hameyer, R. Mertens, U. Pahner, and R. Belmans, "New technique to enhance the accuracy of 2-D/3-D field quantities and forces obtained by standard finite-element solutions," Proc. IEE-Sci. Meas. Technol., vol. 145, no. 2, pp. 67-75, Mar. 1998.

[5] T. Low, C. Bi, and Z. Liu, "A hybrid technique for electromagnetic torque and force analysis of electric machines," COMPEL, Int. J. Comput. Math. Elect. Eng., vol. 16, no. 3, pp. 191-205, 1997.

[6] J. Coulomb and G. Meunier, "Finite element implementation of virtual work principle for magnetic or electric force and torque computation," IEEE Trans. Magn., vol. 20, no. 5, pp. 1894-1896, Sep. 1984.

[7] D. Staton, R. Deodhar, W. Soong, and T. Miller, "Torque prediction using the flux-MMF diagram in ac, dc, and reluctance motors," IEEE Trans. Ind. Appl., vol. 32, no. 1, pp. 180-188, Jan./Feb. 1996.

[8] R. Deodhar, D. Staton, T. Jahns, and T. Miller, "Prediction of cogging torque using the flux-MMF diagram technique," IEEE Trans. Ind. Appl., vol. 32, no. 3, pp. 569-576, May/Jun. 1996.

[9] T. Miller, M. Popescu, C. Cossar, M. McGilp, and J. A. Walker, "Calculating the interior permanent magnet motor", in Conf. Rec. IEEE IEMDC'03, vol. 2, Madison, WI, Jun. 2003, pp. 1181-1187.

[10] T. J. E. Miller and M. I. McGilp, PC-FEA 5.0 for Windows-Software. Glasgow, U.K.: SPEED Lab., Univ. Glasgow, 2002.

[11] S. Williamson and A. Knight, "Performance of skewed single-phase line-start permanent magnet motors," IEEE Trans. Ind. Appl., vol. 35, no. 3, pp. 577-582, May/Jun. 1999.

[12] T. Jahns and W. Soong, "Pulsating torque minimization techniques for permanent magnet ac motor drives-A review," IEEE Trans. Ind. Electron., vol. 43, no. 2, pp. 321-330, Apr. 1996.

[13] L. Parsa, L. Hao, and H. Toliyat, "Optimization of average and $\operatorname{cog}$ ging torque in 3-phase IPM motor drives," in Conf. Rec. IEEE-IAS Annu. Meeting, vol. 1, Pittsburgh, PA, Oct. 2002, pp. 417-424.

[14] A. Arkkio, "Analysis of induction motors based on the numerical solution of the magnetic field and circuit equations," Acta Polytech. Scand. Elect. Eng. Ser., ser. no. 59, 1987.

[15] M. Antila, E. Lantto, and A. Arkkio, "Determination of forces and linearized parameters of radial active magnetic bearings by finite element technique," IEEE Trans. Magn., vol. 34, no. 3, pp. 684-694, May 1998.

[16] W. N. Fu, Z. J. Liu, and C. Bi, "A dynamic model of the disk drive spindle motor and its applications," IEEE Trans. Magn., vol. 38, no. 2, pp. 973-976, Mar. 2002.

[17] M. Marinescu and N. Marinescu, "Numerical computation of torques in permanent magnet motors by Maxwell stresses and energy method," IEEE Trans. Magn., vol. 24, no. 1, pp. 463-466, Jan. 1988.

[18] R. Strahan, "Energy conversion by nonlinear permanent magnet machines," Proc. IEE-Elect. Power Appl., vol. 145, no. 3, pp. 193-198, May 1998.

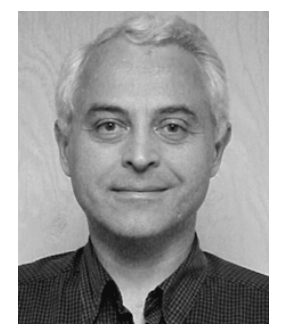

Dan M. Ionel (M'91-SM'01) received the M.Eng. and Ph.D. degrees in electrical engineering from the Polytechnic University of Bucharest, Bucharest, Romania.

Since 2001, he has been a Principal Electromagnetic Engineer with the Corporate Technology Center, A. O. Smith Corporation, Milwaukee, WI. $\mathrm{He}$ began his career with the Research Institute for Electrical Machines (ICPE-ME), Bucharest, Romania, and continued in the U.K., where he was with the SPEED Laboratory at the University of Glasgow and then the Brook Crompton Company, Huddersfield, U.K.. His previous professional experience also includes a one-year Leverhulme visiting fellowship at the University of Bath, U.K. 


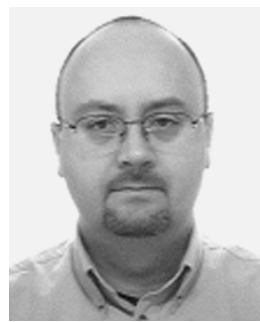

Mircea Popescu (M'98-SM'04) was born in Bucharest, Romania. He received the M.Eng. and Ph.D. degrees from the University "Politehnica" Bucharest, Bucharest, Romania, in 1984 and 1999, respectively, and the D.Sc. degree from Helsinki University of Technology, Espoo, Finland, in 2004, all in electrical engineering.

From 1984 to 1997, he was involved in industrial research and development at the Research Institute for Electrical Machines (ICPE-ME), Bucharest, Romania, as a Project Manager. From 1991 to 1997, he cooperated as a Visiting Assistant Professor with the Electrical Drives and Machines Department, University "Politehnica" Bucharest. From 1997 to 2000, he was a Research Scientist with the Electromechanics Laboratory, Helsinki University of Technology. Since 2000, he has been a Research Associate with the SPEED Laboratory, University of Glasgow, Glasgow, U.K.

Dr. Popescu was the recipient of the 2002 First Prize Paper Award from the Electric Machines Committee of the IEEE Industry Applications Society.

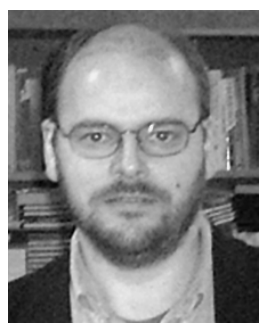

Malcolm I. McGilp was born in Helensburgh, U.K., in 1965. He received the B.Eng. (Hons.) degree in electronic systems and microcomputer engineering from the University of Glasgow, Glasgow, U.K., in 1987.

Since graduating, he has been with the SPEED Laboratory, University of Glasgow, first as a Research Assistant from 1987 to 1996 and as a Research Associate since then. He is responsible for the software architecture of the SPEED motor design software and has developed the interface and user facilities which allow it to be easy to learn and integrate with other PC-based software.

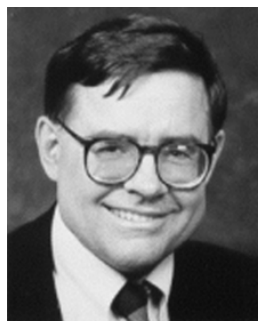

T. J. E. Miller (M'74-SM'82-F'96) is a native of Wigan, U.K. He received the B.Sc. degree from the University of Glasgow, Glasgow, U.K., and the Ph.D. degree from the University of Leeds, Leeds, U.K.

$\mathrm{He}$ is Professor of Electrical Power Engineering and founder and Director of the SPEED Consortium at the University of Glasgow, Glasgow, U.K. He is the author of over 100 publications in the fields of motors, drives, power systems, and power electronics, including seven books. From 1979 to 1986, he was an Electrical Engineer and Program Manager at GE Research and Development, Schenectady, NY, and his industrial experience includes periods with GEC (UK), British Gas, International Research and Development, and a student apprenticeship with Tube Investments Ltd.

Prof. Miller is a Fellow of the Institution of Electrical Engineers, U.K.

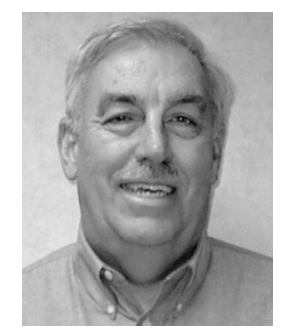

Stephen J. Dellinger received the B.Sc. and M.Sc. degrees in electrical engineering from the University of Dayton, Dayton, $\mathrm{OH}$.

Currently, he is Director of Engineering with the Electric Products Company, A. O. Smith Corporation, Tipp City, $\mathrm{OH}$. His responsibilities include the development and introduction to manufacturing of new motor technologies. He has been with A. O. Smith Corporation for more than 35 years and, during this time, held various positions in manufacturing, engineering, and management. 